\title{
Different Distribution of Pentraxin 3 and C-Reactive Protein in Coronary Atherosclerotic Plaques
}

\author{
Yunosuke Matsuura ${ }^{1,2}$, Kinta Hatakeyama ${ }^{2}$, Takuroh Imamura ${ }^{1}$, Toshihiro Tsuruda $^{1}$, Yoshisato Shibata ${ }^{3}$, \\ Tatsuhiko Kodama ${ }^{4}$, Kazuo Kitamura ${ }^{1}$ and Yujiro Asada ${ }^{2}$ \\ ${ }^{1}$ Department of Internal Medicine, University of Miyazaki, Miyazaki, Japan \\ ${ }^{2}$ Department of Pathology, Faculty of Medicine, University of Miyazaki, Miyazaki, Japan \\ ${ }^{3}$ Miyazaki Medical Association Hospital, Miyazaki, Japan \\ ${ }^{4}$ Laboratory for Systems Biology and Medicine, Research Center for Advanced Science and Technology, University of Tokyo, Japan
}

\begin{abstract}
Aim: To understand the differences between histopathological characteristics related to PTX3 (pentraxin 3) and CRP (C-reactive protein) in coronary atherosclerotic plaques.

Methods and Results: To assess the localization of PTX3 and CRP in coronary plaque, immunohistochemistry was performed using 157 coronary artery specimens from 45 autopsied cases. Overall, immunoreactivity to CRP was more intense than that to PTX3 in lipid rich plaque; however, PTX3 was notably abundant in areas of intraplaque hemorrhage, in which CRP was quite sparse. On quantitative analysis, complicated plaques showed more immunopositive area of PTX3 than fibroatheroma, but with CRP, this trend disappeared. In addition, we examined the phenotype of macrophages in PTX3- and CRP-rich areas using CD163 staining (M2 macrophages). Consequently, these areas were differently characterized by the accumulation of macrophages with high and low magnitude of CD163 positivity, respectively. Next, we immunohistochemically investigated relationships among PTX3, CRP, histological components and clinical presentation in 73 coronary atherectomy specimens obtained from 35 and 38 patients with unstable (UAP) and stable angina pectoris (SAP), respectively. Both PTX3 and CRP were more intense in culprit plaques from patients with UAP than with SAP, and they significantly correlated with CD68 (pan macrophage)-positive areas; however, there was no correlation between PTX3 and CRP.

Conclusion: Although PTX3 and CRP were more enhanced in unstable than in stable coronary plaques, their distribution distinctly differed, suggesting that they play distinct biological roles in unstable plaques.
\end{abstract}

J Atheroscler Thromb, 2012; 19:837-845.

Key words; Pentraxin 3 (PTX3), C-reactive protein (CRP), Coronary artery disease, Intraplaque hemorrhage, Macrophages

\section{Introduction}

Chronic vascular inflammation plays a pivotal role in atherogenesis and plaque instability ${ }^{1)}$. To date, several inflammatory mediators have been implicated in the pathogenesis of cardiovascular disease $(\mathrm{CVD})^{2}$.

Address for correspondence: Kinta Hatakeyama, Department of Pathology, Faculty of Medicine, University of Miyazaki, 5200 Kihara Kiyotake, Miyazaki 889-1692, Japan

E-mail: kpathol@fc.miyazaki-u.ac.jp

Received: November 10, 2011

Accepted for publication: March 22, 2012
Among them, C-reactive protein (CRP) is the most frequently associated with CVD and is widely used in clinical settings due to its potential value as a diagnostic indicator and prognostic predictor of $\mathrm{CVD}^{3-9)}$. CRP is a classic short pentraxin produced mainly in the liver in response to inflammatory mediators, particularly interleukin (IL)- $6^{10)}$. On the other hand, pentraxin 3 (PTX3) is a prototypic long pentraxin synthesized by diverse cell types involved in atherosclerotic lesions, including macrophages, endothelial cells and smooth muscle cells in response to inflammatory stimuli such as IL- $1 \beta$ and tumor necrosis fac- 
tor (TNF) $\alpha^{11,12)}$; therefore, plasma or tissue levels of PTX3 are considered to reflect localized vascular inflammation. Increasing evidence indicates that PTX3 has high diagnostic value for acute myocardial infarction (AMI) and unstable angina pectoris (UAP) ${ }^{13)}$ and that it can serve as a significant prognostic predictor in patients with $\mathrm{CVD}^{14,15)}$.

Both CRP and PTX3 are found in human atherosclerotic plaques ${ }^{16-18)}$. We previously demonstrated that CRP accumulation is enhanced in unstable coronary plaques, suggesting that CRP is associated with plaque instability ${ }^{19}$; however, its relationship to PTX3 in coronary plaques has not yet been examined. Also, regarding the differences between PTX3 and CRP, there is little histological evidence of their effect on coronary atherosclerotic plaques. Therefore, we investigated, 1) the localization and quantitative distribution of PTX3 and CRP in various stages of coronary atherosclerotic lesions, 2) whether the extent of PTX3 in coronary culprit plaque is actually associated with clinical presentation, and 3) the nature of the association between histological components and PTX3 accumulation in culprit coronary plaques.

\section{Materials and Methods}

\section{Materials}

All coronary artery specimens were collected under a study protocol approved by the institutional review board of the University of Miyazaki. Details of the specimens are as follows: 157 coronary artery specimens were obtained from 45 autopsies and 73 culprit coronary plaques were obtained by directional coronary atherectomy (DCA) for a de novo lesion from patients with UAP $(n=35)$ and SAP $(n=38)$. Postmortem coronary arteries were also removed as described $^{20)}$ and Table $\mathbf{1}$ shows the profiles of autopsy cases. Table 2 lists the clinical characteristics of the patients who had undergone the DCA procedure. Patients with apparent infectious diseases, malignancies or chronic inflammatory diseases were excluded from this group. Risk factors for coronary artery disease and medications upon admission except for aspirin did not statistically differ between patients with UAP and those with SAP. Culprit lesions were comprehensively identified by electrocardiography, coronary angiography and by assessing left ventricle wall motion abnormalities using left ventriculography or echocardiography. All coronary artery specimens were fixed in 4\% paraformaldehyde and embedded in paraffin, and tissue sections were stained with hematoxylin and eosin (HE) for morphological studies. The postmortem specimens were histologically categorized
Table 1. Profiles of autopsied cases

\begin{tabular}{lc}
\hline Number & 45 \\
Age $(\mathrm{y} ;$ mean \pm SD) & $63.1 \pm 13.8$ \\
Male, n $(\%)$ & $35(78)$ \\
Cardiovascular death, n (\%) & $18(40)$ \\
Acute myocardial infarction, $\mathrm{n}$ & 8 \\
Non-cardiovascular death, $\mathrm{n}(\%)$ & $27(60)$ \\
\hline
\end{tabular}

according to the American Heart Association (AHA) classification $^{21,22)}$ (Table 3).

\section{Immunohistochemistry}

Serial sections (4- $\mu \mathrm{m}$ thick) were immunohistochemically stained using the primary antibodies mentioned below ${ }^{19)}$. In brief, after blocking endogenous peroxidase activity in 3\% hydrogen peroxide in methanol for 20 minutes, the sections were incubated with primary monoclonal antibodies to PTX3 (clone PPZ 1228; Perseus Proteomics Inc., Tokyo, Japan), CRP (clone CRP-8; Sigma, St. Louis, MO, USA), macrophages (CD68: clone PGM-1; Dako, Glostrup, Denmark, and CD163: clone 10D6; Leica Biosystems, Newcastle, UK), smooth muscle cells ( $\alpha$-smooth muscle actin (SMA); Dako) and T lymphocyte (CD3: clone F7.2.38; Dako). Antigen retrieval was performed for PTX3, CD163 and CD3 staining using a microwave, and then the sections were immunostained using the EnVision + kit (Dako). Horseradish peroxidase activity was visualized with 3 , 3' - diaminobenzidine containing hydrogen peroxide. The negative control contained a normal mouse IgG instead of the primary antibody.

\section{Detection of Iron Deposition}

Iron deposition was detected using Perl's iron staining.

\section{Morphometric Image Analysis}

Specimens were quantified using a color imaging analysis system (Win ROOF; Mitani, Fukui, Japan). In postmortem coronary specimens, we identified 6 high intensity areas of PTX3 or CRP through an entire coronary plaque, quantified the immunopositive area per image at $\times 400$ magnification, and then assessed the mean rate (\%) in each section. In atherectomy specimens, we quantified immunopositive areas and iron deposition areas as previously described ${ }^{23,24)}$.

$\mathrm{T}$ lymphocytes defined by $\mathrm{CD} 3$ were counted at $\times 400$ magnification and their density is expressed as numbers per $\mathrm{mm}^{2}$. Two investigators (Y.A. and K. H.) who were blinded to the clinical classification of the 
Table 2. Clinical characteristics of patients who underwent Directional Coronary Atherectomy (DCA)

\begin{tabular}{|c|c|c|c|}
\hline & $\mathrm{UAP}(n=35)$ & $\operatorname{SAP}(n=38)$ & $p$ value \\
\hline Age $(y)$ & $61.8 \pm 1.9$ & $63.0 \pm 1.8$ & 0.62 \\
\hline Male, n (\%) & $27(77)$ & $33(87)$ & 0.36 \\
\hline Body mass index $\left(\mathrm{kg} / \mathrm{m}^{2}\right)$ & $24.7 \pm 0.7$ & $24.9 \pm 0.6$ & 0.59 \\
\hline Hypertension, n (\%) & $25(71)$ & $26(68)$ & 0.80 \\
\hline Dyslipidemia, n (\%) & $18(51)$ & $21(55)$ & 0.81 \\
\hline Diabetes mellitus, n (\%) & $14(40)$ & $15(39)$ & 1.00 \\
\hline Smoking, n (\%) & $11(31)$ & $19(50)$ & 0.15 \\
\hline Family history, n (\%) & $7(20)$ & $8(21)$ & 1.00 \\
\hline Aspirin, n (\%) & $28(80)$ & $37(97)$ & 0.02 \\
\hline ACE-I/ARB, n (\%) & $13(37)$ & $16(42)$ & 0.81 \\
\hline Statins, n (\%) & $8(23)$ & $12(32)$ & 0.44 \\
\hline $\mathrm{CCB}, \mathrm{n}(\%)$ & $16(46)$ & $17(45)$ & 1.00 \\
\hline Beta blocker, n (\%) & $5(14)$ & $7(18)$ & 0.76 \\
\hline
\end{tabular}

ACE-I, Angiotensin-converting enzyme inhibitor; ARB, Angiotensin II type I receptor blocker; CCB, Calcium channel blocker.

patients performed all morphometric analyses.

\section{Statistical Analysis}

Data were analyzed using JMP 7.0 (SAS Institute Inc., Cary, NC) and Graph Pad Prism 5.0 (GraphPad Software Inc., San Diego, CA). Values are expressed as the means with standard deviation (SD) or as medians with interquartile ranges (IQR) when the variance was skewed. Categorical variables were compared using Fisher's exact test. Differences in histological composition among groups were compared using the MannWhitney $U$ test or the Kruskal-Wallis test followed by Dunn's multiple comparison post-hoc analysis. Relationships among histological characteristics were evaluated using Spearman's correlation test. A $p$-value of $<0.05$ was considered significant.

\section{Results}

\section{Immunoreactivity for PTX3 and CRP in Coronary Plaques}

In both sets of coronary specimens, immunoreactivity for PTX3 and CRP was observed mainly as depositions in the extracellular matrix, and as expression in some macrophages and smooth muscle cells.

\section{Different Quantitative Distributions between PTX3 and CRP Among the Types of Coronary Atherosclerotic Lesions}

Quantitative analysis of PTX3- and CRP-positive areas among the types of atherosclerotic lesions was investigated using serial sections of the postmortem coronary artery (Fig. 1). Both PTX3 (Fig. 1A) and CRP (Fig. 1B) are more abundant in advanced
Table 3. Classification of 157 coronary artery specimens

\begin{tabular}{ll}
\hline \multicolumn{1}{c}{ AHA classification } & $\mathrm{n}$ \\
\hline Type II-III (Fatty streak, preatheroma) & 17 \\
Type IV (Atheroma) & 23 \\
Type Vc (Fibrous plaque) & 22 \\
Type Va (Fibro-atheroma) & 33 \\
Type VI (Complicated lesion/Ruptured plaque) & 62 \\
\hline
\end{tabular}

atherosclerotic lesions than in early lesions. In advanced lesions, both PTX3 and CRP were more accumulated in fibroatheroma $(\mathrm{Va})$ and complicated lesions (VI) than in fibrous plaque (Vc). CRP but not PTX3 was more augmented in fibroatheroma than in atheroma (IV). On the other hand, PTX3 but not $\mathrm{CRP}$ was more enhanced in complicated lesions than in fibroatheroma.

\section{Different Localization between PTX3 and CRP in Coronary Plaques}

Immunohistochemistry using serial sections showed that PTX3 and CRP were distinctly localized in coronary atherosclerotic lesions. Immunoreactivity for PTX3 was quite sparse in lipid-rich lesions in fibroatheroma (Fig. 2A and B), whereas that for CRP was particularly intense surrounding and within the lipid core (Fig. 2C). Areas with intraplaque hemorrhage (type VI lesions, Fig. $\mathbf{3 A}$ and $\mathbf{B}$ ) contained abundant PTX3, but little CRP (Fig. 3C).

In postmortem coronary arteries, 39 fibroatheromatous plaques had intraplaque hemorrhage in 62 complicated lesions. Fig. 4 shows the quantitative analysis of PTX3- and CRP-positive areas between 
A

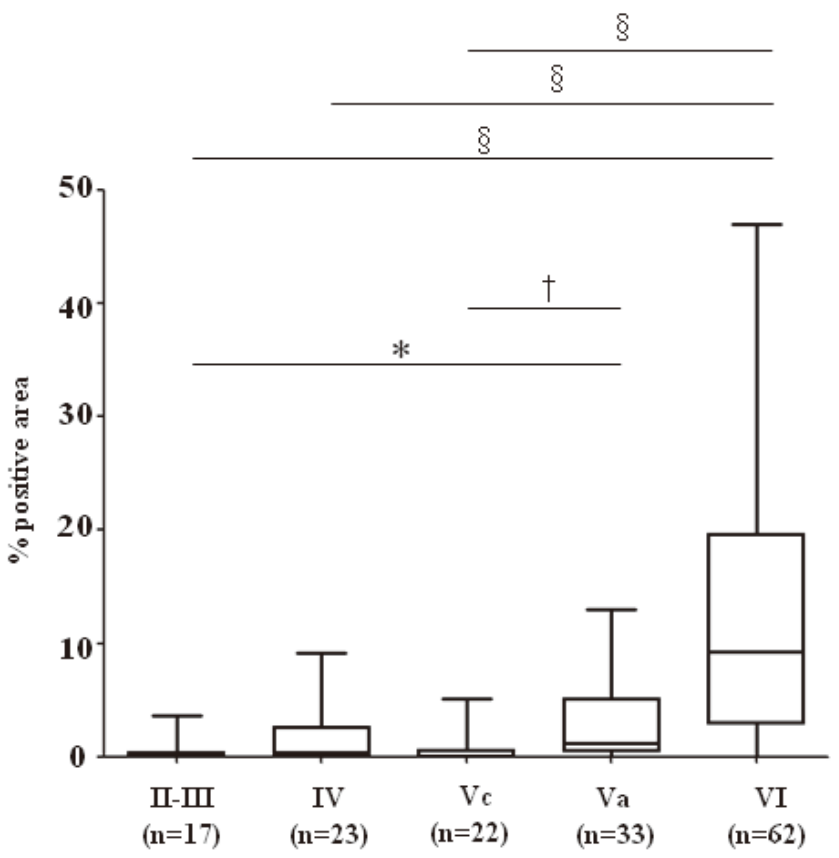

B

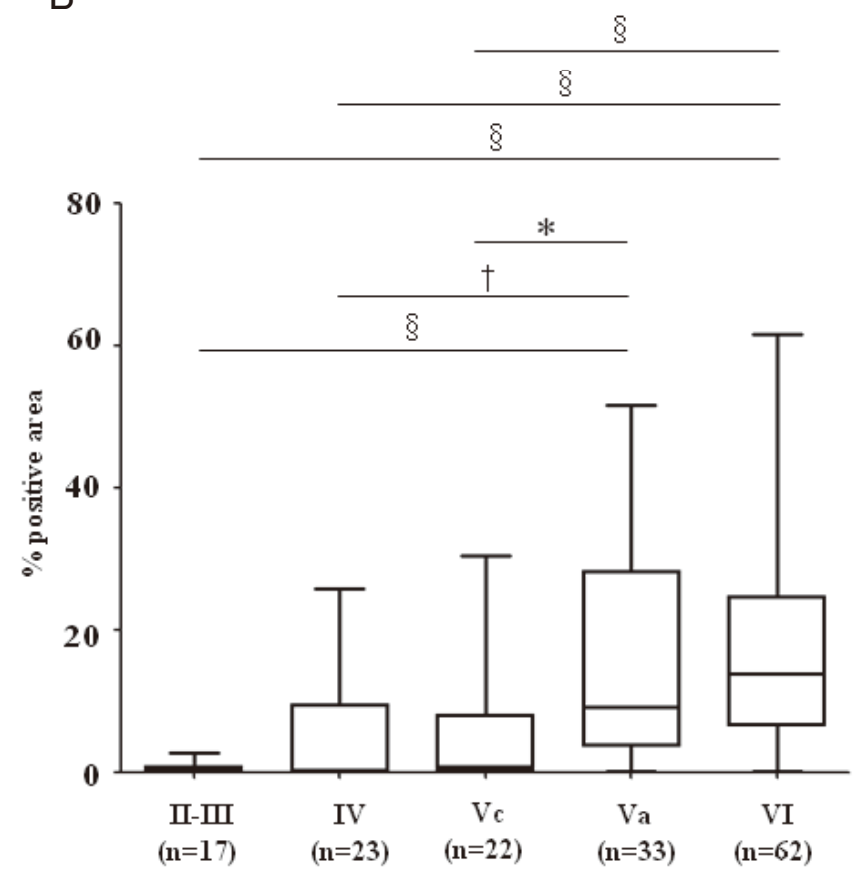

Fig. 1. Quantitative analysis of PTX3- and CRP-positive areas among the types of atherosclerotic lesions according to AHA classification (A: PTX3, B: CRP)

fibroatheromatous plaques with and without intraplaque hemorrhage. Immunopositive areas for PTX3 but not CRP were significantly greater in fibroatheroma with hemorrhage (complicated lesion) compared to fibroatheroma without hemorrhage.

\section{PTX3 Closely Localized CD163-Positive Macrophages}

In both PTX3- and CRP-rich areas, the accumulation of macrophages was defined as CD68-positive cells (Fig. 2E and 3E); however, PTX3- and CRP-rich areas were differently characterized by the accumulation of macrophages with high and low magnitude of CD163 positivity, respectively (Fig.3D and 2D).

\section{Enhanced Accumulation of PTX3 and CRP in Culprit Coronary Plaques from Patients with UAP than SAP, but not Significant Correlation between PTX3 and CRP}

Table 4 compares the histological characteristics of culprit coronary plaques between UAP and SAP. Quantitative analysis indicated that PTX3 and CRP were more intense in culprit coronary plaques from patients with UAP than with SAP. In addition, immunopositive areas of CD68 (pan macrophages) significantly correlated with those of both PTX3 and CRP $(r=0.31, p=0.007$ and $r=0.34, p=0.003$, respectively), whereas the number of CD3-positive cells ( $\mathrm{T}$ lymphocytes) significantly correlated only with CRP $(r=0.27, p=0.02)$. Between PTX3 and CRP, there was no significant correlation $(r=0.21, p=n . s)$.

PTX3 but not CRP Significantly Correlates with the Extent of CD163 and Iron Deposition in Atherectomy Specimens from Patients with UAP

In culprit plaques obtained from patients with UAP, we examined the correlation between immunopositive areas of CD163 and the extent of iron deposition with PTX3 and CRP, respectively. As a result, the extent of both CD163 and iron deposition significantly correlated with those of PTX3 $(r=0.35$, $p=0.037, r=0.42, p=0.011$, respectively) but not with those of CRP $(r=0.09, p=0.61, r=0.18, p=$ 0.29 , respectively).

\section{Discussion}

The major findings of the present study are as follows: 1) PTX3 but not CRP was augmented in complicated plaque compared to fibroatheroma, whereas CRP but not PTX3 was more accumulated in fibroatheroma than in atheroma; 2) The localization 

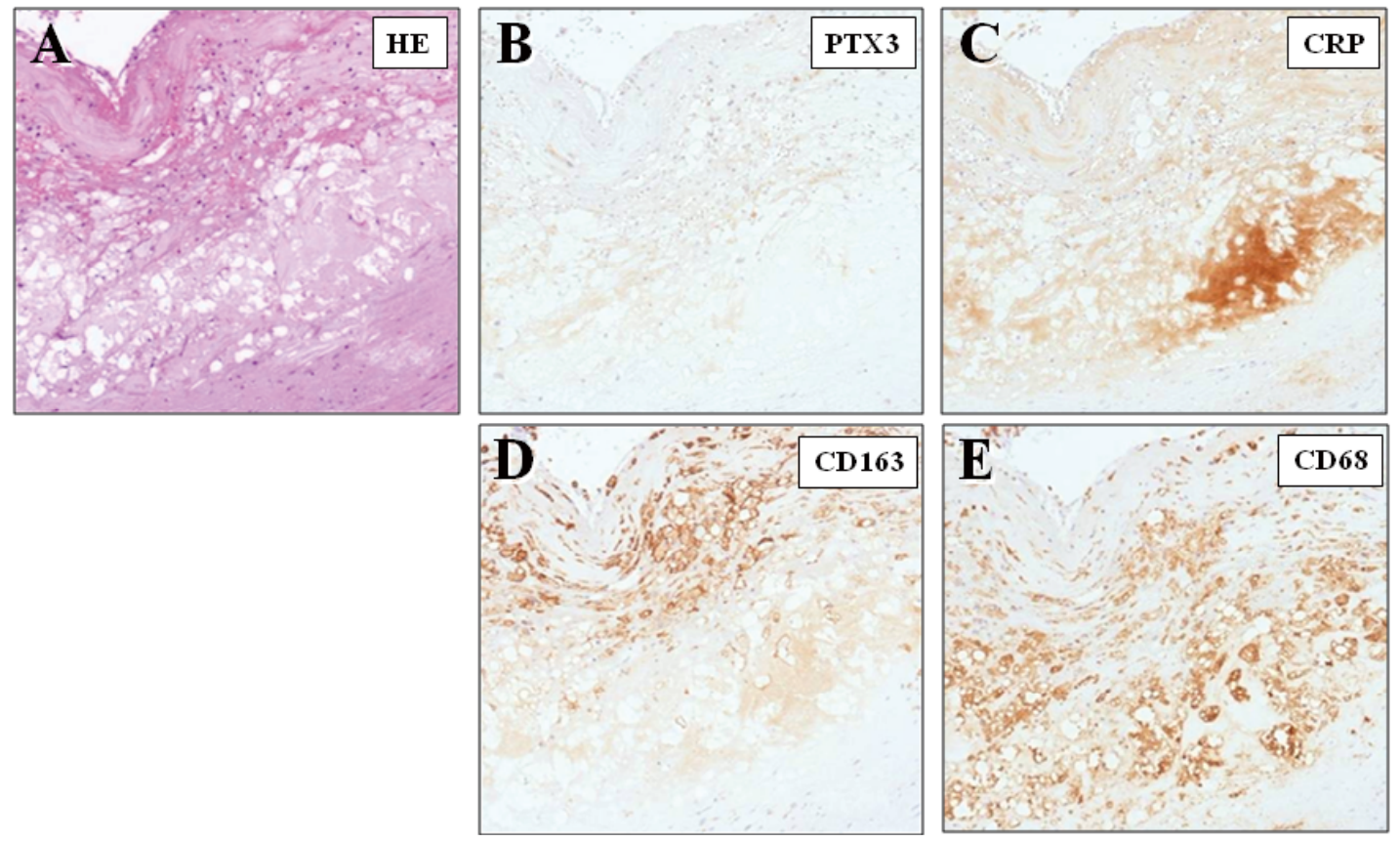

Fig. 2. Representative microphotographs of fibroatheromatous lesion in coronary plaque from an autopsied case. Lipid and macrophage-rich plaque covered with fibrous cap (A, HE stain). Immunoreactivity for PTX3 (B) is sparse whereas that for CRP (C) is abundant in plaque, especially at lipid core. CD163 (D) positive macrophages (E) mainly localize beneath the cap and not in the lipid core. Original magnification $\times 100$
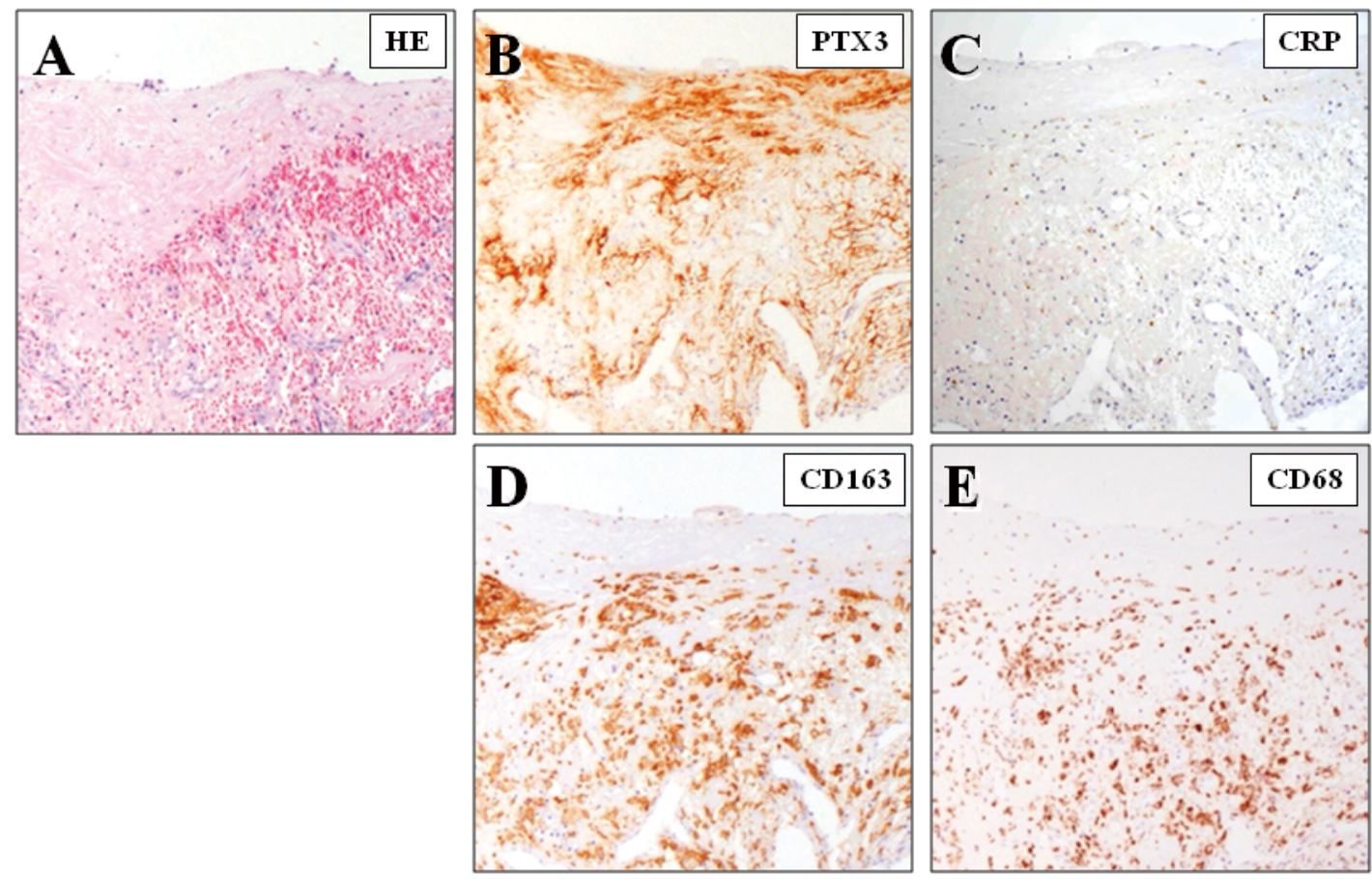

Fig. 3. Representative microphotographs of complicated lesion with intraplaque hemorrhage of coronary plaque from an autopsied case. PTX3 immunoreactivity is abundant at hemorrhagic site (A, B), whereas that of CRP is sparse (C). Many macrophages (E) are CD163 positive (D). Original magnification $\times 100$ 

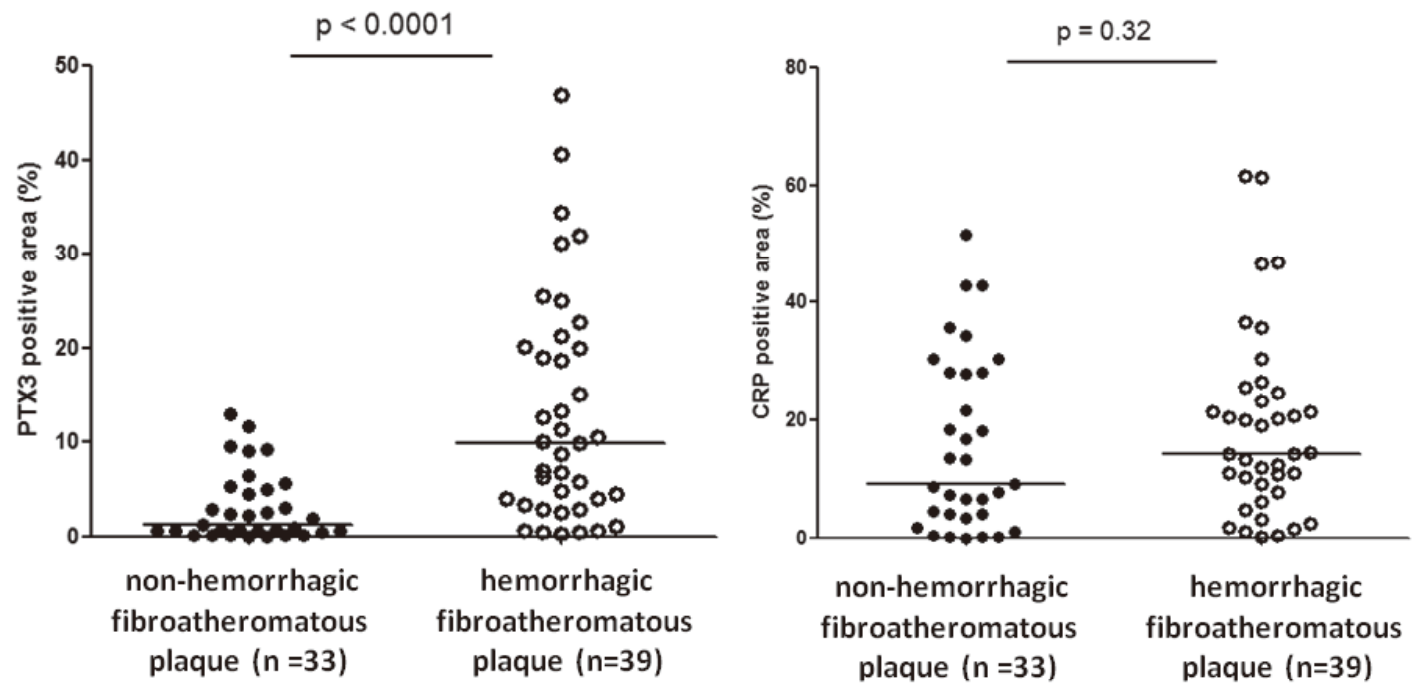

Fig.4. Quantitative analysis of PTX3- and CRP-positive areas in fibroatheromatous plaques with or without intraplaque hemorrhage. Immunopositive areas for PTX3 but not CRP were significantly greater in fibroatheroma with hemorrhage (complicated lesion) than in fibroatheroma without hemorrhage.

of PTX3 differed from that of CRP in coronary plaque, and macrophages closely located with these molecules exhibited different phenotypes; 3) Abundant PTX3 was observed in areas of intraplaque hemorrhage, but CRP was scarce in such areas; 4) PTX3 as well as CRP was more enhanced in unstable than in stable coronary plaque and both PTX3 and CRP were significantly correlated with the amplitude of macrophages; however, there was no significant correlation between PTX3 and CRP.

PTX3 and CRP belong to the pentraxin family, and recent studies indicated that both are associated with CVD; however, little is understood about the differences regarding the aspects of human atherosclerotic lesions associated with PTX3 and CRP. Therefore, in the clinical setting, it is still difficult to use them as biomarkers for the detection or stratification of high-risk coronary plaques.

Regarding the immunoreactivity of PTX3 and CRP, our results are consistent with previous studies ${ }^{16-18)}$; however, it remains unclear whether the localizations of two pentraxins in coronary plaques show a similar or different trend. The present study showed that the localization of PTX3 is obviously different from that of CRP in both sets of coronary specimens, and also the amounts of two pentraxins in coronary plaques differed depend on the types of atherosclerosis, although both pentraxins were enhanced with unstable coronary plaque.

The analysis of postmortem coronary specimens showed that PTX3 could stratify complicated plaques from other types, but CRP showed similar levels between fibroatheroma and complicated plaques, suggesting that PTX3 is superior to CRP as a biomarker in the detection of complicated plaques.

On the other hand, CRP but not PTX3 seems to be associated with development from atheroma to fibroatheroma, although the underlying mechanism is unknown.

Taken together, the two pentraxins are associated with different characteristics in unstable coronary plaques, suggesting that their combination might allow the appropriate stratification of high-risk coronary plaque.

It is difficult to explain the underlying grounds for these different localizations of two pentraxins in coronary plaques because vascular cells are exposed to a multiplicity of signals.

Several differences have been demonstrated between ligands with which PTX3 and CRP inter$\mathrm{act}^{25)}$. In the present study, PTX3 could be observed mainly as extracellular matrix deposition and its localization was different from that of CRP. This finding might be due to the spatial distribution of extracellular ligands in atherosclerotic plaques to which two pentraxins could bind. Previous studies have demonstrated that PTX3 plays an important role in extracellular matrix (ECM) remodeling and organization in cumulus oophorus ${ }^{26)}$ or inflamed tissues ${ }^{27)}$, in which it was suggested that PTX3 interacts with tumor 
Table 4. Comparison of histological characteristics between UAP and SAP

\begin{tabular}{lccc}
\hline & UAP $(n=35)$ & SAP $(n=38)$ & $p$ value \\
\hline CD68 (\% positive area) & $3.57(0.96-7.42)$ & $0.97(0.27-3.10)$ & 0.001 \\
CD3 (number per mm $\left.{ }^{2}\right)$ & $24(13-67)$ & $9(2-35)$ & 0.005 \\
$\alpha$-SMA (\% positive area) & $7.98(2.68-11.42)$ & $8.89(3.80-14.34)$ & 0.23 \\
PTX3 (\% positive area) & $1.24(0.24-5.96)$ & $0.43(0.08-1.62)$ & 0.01 \\
CRP (\% positive area) & $1.01(0.14-3.85)$ & $0.08(0-1.07)$ & 0.002 \\
\hline
\end{tabular}

necrosis factor $\alpha$-induced protein 6 (TSG-6), which is a ligand of PTX3 but not CRP and has anti-inflammatory properties ${ }^{28)}$. Although it remains unclear how the interaction between TSG-6 and PTX3 contributes to extracellular matrix organization in atherosclerotic plaque, this might be one possible explanation for the different localization between the two pentraxins.

Insights from macrophage polarities or their framework might help in the interpretation of our observational findings. Emerging evidence showed that macrophage polarities are implicated in various situations of human pathophysiology, such as inflammation, cancer and atherosclerosis, indicating that macrophages seem to be two prominent types of populations, such as M1 (inflammatory) and M2 (antiinflammatory) phenotypes.

CD163 is a specific haemoglobin scavenger receptor that is expressed by resident tissue macrophages $^{29)}$, its expression in macrophages has been associated with anti-inflammatory properties, and therefore it is known to be a marker of M2 macrophage phenotypes. Our results showed that in advanced coronary plaques without complicated lesions, CD163positive macrophages were observed mainly in superficial regions and less in deep layers of plaques. This distribution of CD163 macrophages shows a similar trend to that of PTX3 and is opposite that of CRP. Also, complicated plaques with hemorrhage contained abundant CD163-positive macrophages, which showed high immunoreactivity to PTX3 but not CRP. Yunoki et al. ${ }^{30)}$ found a positive association between intraplaque haemorrhage and CD163 positivity in macrophages in human unstable coronary plaques. Boyle et al. ${ }^{31)}$ indicated that CD163-positive macrophages are distinct from pro-inflammatory lipid core macrophages characterized by high HLA-DR. Additionally, CD163 expression in monocytes and macrophages was markedly induced by anti-inflammatory cytokines such as IL-10 and inhibited by pro-inflammatory cytokines such as interferon (IFN) $-\gamma$. PTX3 expression in dendritic cells and monocytes is similarly stimulated by IL-10 and inhibited by IFN $-y^{32,33)}$. These lines of evidence suggest a cross-relationship between PTX3- and CD163-positive macrophages in atherosclerotic plaques. Furthermore, recent vitro studies indicated that T-lymphocytes incubated with CRP-pulsed dendritic cells displayed increased IFN-y secretion and proliferation ${ }^{34)}$ and that CRP polarized human monocyte differentiation toward M1 macrophages and also prevented the polarization of IL- 4 induced M2 macrophage phenotypes ${ }^{35)}$. These results might support our findings that, in atherectomy specimens, CRP but not PTX3 is significantly correlated with T-lymphocytes and also, the CRP-rich area in post-mortem coronary plaques could be characterized by the magnitude of CD163-negative macrophages.

Recent reports showed that PTX3 deficiency in mice is associated with an increased burden of vascular lesions as well as macrophage accumulation within atherosclerotic lesions ${ }^{36)}$. Taken together, PTX3 has potential anti-atherogenic properties and is amplified in various involved cells, especially in complicated atherosclerotic lesions. Our observation that macrophages closely localized with PTX3 but not with CRP supports the notion of an athero-protective role for PTX3.

The underlying mechanisms and interactions between PTX3/CRP and macrophage phenotypes remain to be clarified in further studies.

\section{Study Limitations}

The present study has several limitations. We excluded patients with an apparent inflammatory condition and malignancies from among those who had undergone the DCA procedure; however, we examined postmortem specimens with inflammatory conditions due to organ failure and malignancies. Secondly, fewer patients with UAP than with SAP received aspirin upon admission before undergoing DCA and therefore, the anti-inflammatory effect of this agent might have influenced PTX3 and CRP expression in coronary plaques; however, all patients received aspirin at the time of the DCA procedure, and areas that were immunopositive for PTX3 and $\mathrm{CRP}$ in coronary plaque did not significantly differ 
regardless of whether aspirin was administered at the time of admission (data not shown). Finally, a quantitative relationship between serum and tissue PTX3 and CRP could not be assessed because blood samples from the patients were unavailable.

In conclusion, both PTX3 and CRP were more enhanced in unstable coronary plaque; however, they were differently localized in coronary plaque and differently distributed among types of atherosclerosis, suggesting that PTX3 and CRP reflect different aspects of unstable coronary plaque. The two pentraxins might play different roles in the pathogenesis of plaque progression and in the instability of coronary artery disease.

\section{Acknowledgements and Notice of Grant Support}

This work was supported in part by Grants-inAid for Scientific Research in Japan (Nos. 20590344, 23390084) from the Ministry of Education, Science, Sports and Culture of Japan.

\section{Conflict of Interest}

None declared.

\section{References}

1) Libby P: Inflammation in atherosclerosis. Nature, 2002; 420: 868-874

2) Libby P, Ridker PM, Hansson GK: Inflammation in atherosclerosis: from pathophysiology to practice. J Am Coll Cardiol, 2009; 54: 2129-2138

3) Corrado E, Rizzo M, Coppola G, Fattouch K, Novo G, Marturana I, Ferrara F, Novo S: An update on the role of markers of inflammation in atherosclerosis. J Atheroscler Thromb, 2010; 17: 1-11

4) Homma Y: Predictors of atherosclerosis. J Atheroscler Thromb, 2004; 11: 265-270

5) Anand SS, Razak F, Yi Q, Davis B, Jacobs R, Vuksan V, Lonn E, Teo K, McQueen M, Yusuf S: C-reactive protein as a screening test for cardiovascular risk in a multiethnic population. Arterioscler Thromb Vasc Biol, 2004; 24: 1509-1515

6) Buckley DI, Fu R, Freeman M, Rogers K, Helfand M: $\mathrm{C}$-reactive protein as a risk factor for coronary heart disease: a systematic review and meta-analyses for the U.S. Preventive Services Task Force. Ann Intern Med, 2009; 151: 483-495

7) Ray KK, Cannon CP, Cairns R, Morrow DA, Ridker PM, Braunwald E: Prognostic utility of apoB/AI, total cholesterol/HDL, non-HDL cholesterol, or hs-CRP as predictors of clinical risk in patients receiving statin therapy after acute coronary syndromes: results from PROVE ITTIMI 22. Arterioscler Thromb Vasc Biol, 2009; 29: 424-
430

8) Ridker PM, Cannon CP, Morrow D, Rifai N, Rose LM, McCabe CH, Pfeffer MA, Braunwald E; Pravastatin or Atorvastatin Evaluation and Infection Therapy-Thrombolysis in Myocardial Infarction 22 (PROVE IT-TIMI 22) Investigators: C-reactive protein levels and outcomes after statin therapy. N Engl J Med, 2005; 352: 20-28

9) Ferreirós ER, Boissonnet CP, Pizarro R, Merletti PF, Corrado $\mathrm{G}$, Cagide A, Bazzino OO: Independent prognostic value of elevated $\mathrm{C}$-reactive protein in unstable angina. Circulation, 1999; 100: 1958-1963

10) Castell JV, Gómez-Lechón MJ, David M, Fabra R, Trullenque R, Heinrich PC: Acute-phase response of human hepatocytes: regulation of acute-phase protein synthesis by interleukin-6. Hepatology, 1990; 12: 1179-1186

11) Presta M, Camozzi M, Salvatori G, Rusnati M: Role of the soluble pattern recognition receptor PTX3 in vascular biology. J Cell Mol Med, 2007; 11: 723-738

12) Mantovani A, Garlanda C, Doni A, Bottazzi B: Pentraxins in innate immunity: from $\mathrm{C}$-reactive protein to the long pentraxin PTX3. J Clin Immunol, 2008; 28: 1-13

13) Inoue $K$, Sugiyama $A$, Reid PC, Ito $Y$, Miyauchi K, Mukai S, Sagara M, Miyamoto K, Satoh H, Kohno I, Kurata T, Ota H, Mantovani A, Hamakubo T, Daida H, Kodama T: Establishment of a high sensitivity plasma assay for human pentraxin3 as a marker for unstable angina pectoris. Arterioscler Thromb Vasc Biol, 2007; 27: 161-167

14) Latini R, Maggioni AP, Peri G, Gonzini L, Lucci D, Mocarelli P, Vago L, Pasqualini F, Signorini S, Soldateschi D, Tarli L, Schweiger C, Fresco C, Cecere R, Tognoni G, Mantovani A; Lipid Assessment Trial Italian Network (LATIN) Investigators: Prognostic significance of the long pentraxin PTX3 in acute myocardial infarction. Circulation, 2004; 110: 2349-2354

15) Jenny NS, Arnold AM, Kuller LH, Tracy RP, Psaty BM: Associations of pentraxin 3 with cardiovascular disease and all-cause death: the Cardiovascular Health Study. Arterioscler Thromb Vasc Biol, 2009; 29: 594-599

16) Sun H, Koike T, Ichikawa T, Hatakeyama K, Shiomi M, Zhang B, Kitajima S, Morimoto M, Watanabe T, Asada Y, Chen YE, Fan J: C-reactive protein in atherosclerotic lesions: its origin and pathophysiological significance. Am J Pathol, 2005; 167: 1139-1148

17) Rolph MS, Zimmer S, Bottazzi B, Garlanda C, Mantovani A, Hansson GK: Production of the long pentraxin PTX3 in advanced atherosclerotic plaques. Arterioscler Thromb Vasc Biol, 2002; 22: e10-e14

18) Savchenko A, Imamura M, Ohashi R, Jiang $S$, Kawasaki T, Hasegawa G, Emura I, Iwanari H, Sagara M, Tanaka T, Hamakubo T, Kodama T, Naito M: Expression of pentraxin 3 (PTX3) in human atherosclerotic lesions. J Pathol, 2008; 215: 48-55

19) Ishikawa $T$, Hatakeyama $K$, Imamura $T$, Date $H$, Shibata Y, Hikichi Y, Asada Y, Eto T: Involvement of C-reactive protein obtained by directional coronary atherectomy in plaque instability and developing restenosis in patients with stable or unstable angina pectoris. Am J Cardiol, 2003; 91: 287-292

20) Sato Y, Hatakeyama K, Yamashita A, Marutsuka K, Sumiyoshi A, Asada Y: Proportion of fibrin and platelets differs 
in thrombi on ruptured and eroded coronary atherosclerotic plaques in humans. Heart, 2005; 91: 526-530

21) Stary HC, Chandler AB, Glagov $S$, Guyton JR, Insull W Jr, Rosenfeld ME, Schaffer SA, Schwartz CJ, Wagner WD, Wissler RW: A definition of initial, fatty streak, and intermediate lesions of atherosclerosis. A report from the Committee on Vascular Lesions of the Council on Arteriosclerosis, American Heart Association. Arterioscler Thromb, 1994; 14: 840-856

22) Stary HC, Chandler AB, Dinsmore RE, Fuster V, Glagov S, Insull W Jr, Rosenfeld ME, Schwartz CJ, Wagner WD, Wissler RW: A definition of advanced types of atherosclerotic lesions and a histological classification of atherosclerosis. A report from the Committee on Vascular Lesions of the Council on Arteriosclerosis, American Heart Association. Arterioscler Thromb Vasc Biol, 1995; 15: 15121531

23) Nishihira $K$, Imamura $T$, Yamashita $A$, Hatakeyama $K$, Shibata Y, Nagatomo Y, Date H, Kita T, Eto T, Asada Y: Increased expression of interleukin-10 in unstable plaque obtained by directional coronary atherectomy. Eur Heart J, 2006; 27: 1685-1689

24) Solem J, Levin M, Karlsson T, Grip L, Albertsson P, Wiklund O: Composition of coronary plaques obtained by directional atherectomy in stable angina: its relation to serum lipids and statin treatment. J Intern Med, 2006; 259: 267-275

25) Garlanda C, Bottazzi B, Bastone A, Mantovani A: Pentraxins at the crossroads between innate immunity, inflammation, matrix deposition, and female fertility. Annu Rev Immunol, 2005; 23: 337-366

26) Bottazzi B, Bastone A, Doni A, Garlanda C, Valentino S, Deban L, Maina V, Cotena A, Moalli F, Vago L, Salustri A, Romani L, Mantovani A: The long pentraxin PTX3 as a link among innate immunity, inflammation, and female fertility. J Leukoc Biol, 2006; 79: 909-912

27) van Rossum AP, Pas HH, Fazzini F, Huitema MG, Limburg PC, Jonkman MF, Kallenberg CG: Abundance of the long pentraxin PTX3 at sites of leukocytoclastic lesions in patients with small-vessel vasculitis. Arthritis Rheum, 2006; 54: 986-991
28) Maina V, Cotena A, Doni A, Nebuloni M, Pasqualini F, Milner CM, Day AJ, Mantovani A, Garlanda C: Coregulation in human leukocytes of the long pentraxin PTX3 and TSG-6. J Leukoc Biol, 2009; 86: 123-132

29) Graversen JH, Madsen M, Moestrup SK: CD163: a signal receptor scavenging haptoglobin-hemoglobin complexes from plasma. Int J Biochem Cell Biol, 2002; 34: 309-314

30) Yunoki K, Naruko T, Komatsu R, Ehara S, Shirai N, Sugioka K, Nakagawa M, Kitabayashi C, Ikura Y, Itoh A, Kusano K, Ohe T, Haze K, Becker AE, Ueda M: Enhanced expression of haemoglobin scavenger receptor in accumulated macrophages of culprit lesions in acute coronary syndromes. Eur Heart J, 2009; 30: 1844-1852

31) Boyle JJ, Harrington HA, Piper E, Elderfield K, Stark J, Landis RC, Haskard DO: Coronary intraplaque hemorrhage evokes a novel atheroprotective macrophage phenotype. Am J Pathol, 2009; 174: 1097-1108

32) Doni A, Michela M, Bottazzi B, Peri G, Valentino S, Polentarutti N, Garlanda C, Mantovani A: Regulation of PTX3, a key component of humoral innate immunity in human dendritic cells: stimulation by IL-10 and inhibition by IFN-gamma. J Leukoc Biol, 2006; 79: 797-802

33) Polentarutti N, Picardi G, Basile A, Cenzuales S, Rivolta A, Matteucci C, Peri G, Mantovani A, Introna M: Interferon-gamma inhibits expression of the long pentraxin PTX3 in human monocytes. Eur J Immunol, 1998; 28: 496-501

34) Van Vré EA, Bult H, Hoymans VY, Van Tendeloo VF, Vrints CJ, Bosmans JM: Human C-reactive protein activates monocyte-derived dendritic cells and induces dendritic cell-mediated T-cell activation. Arterioscler Thromb Vasc Biol, 2008; 28: 511-518

35) Devaraj S, Jialal I. C-reactive protein polarizes human macrophages to an M1 phenotype and inhibits transformation to the M2 phenotype. Arterioscler Thromb Vasc Biol, 2011; 31: 1397-1402

36) Norata GD, Marchesi P, Pulakazhi Venu VK, Pasqualini F, Anselmo A, Moalli F, Pizzitola I, Garlanda C, Mantovani A, Catapano AL: Deficiency of the long pentraxin PTX3 promotes vascular inflammation and atherosclerosis. Circulation, 2009; 120: 699-708 